\title{
THE ALGEBRA OF REWRITING FOR PRESENTATIONS OF INVERSE MONOIDS
}

\author{
N.D. GILBERT AND E.A. MCDOUGALL \\ Department of Mathematics and the Maxwell Institute for the Mathematical Sciences, \\ Heriot-Watt University, Edinburgh, EH14 4AS
}

\begin{abstract}
We describe a formalism, using groupoids, for the study of rewriting for presentations of inverse monoids, that is based on the Squier complex construction for monoid presentations. We introduce the class of pseudoregular groupoids, an example of which now arises as the fundamental groupoid of our version of the Squier complex. A further key ingredient is the factorisation of the presentation map from a free inverse monoid as the composition of an idempotent pure map and an idempotent separating map. The relation module of a presentation is then defined as the abelianised kernel of this idempotent separating map. We then use the properties of idempotent separating maps to derive a free presentation of the relation module. The construction of its kernel - the module of identities - uses further facts about pseudoregular groupoids.
\end{abstract}

\section{INTRODUCTION}

Inverse semigroups (and inverse monoids) comprise a class of algebraic structures that sit naturally between the class of semigroups and the class of groups, and are the natural candidates for semigroups that are structurally closest to groups. However, inverse semigroup presentations do not sit quite so naturally between semigroup presentations and group presentations, but have particular features that set them apart. For example, a finitely generated free inverse semigroup is not finitely presented as a semigroup [24], does not have a regular language of normal forms [8], and no free inverse monoid has context-free word problem [1].

In this paper we consider presentations of inverse monoids as rewriting systems, and attempt to replicate the formalism for describing rewriting in monoid presentations due to Squier [25, 26], and for group presentations due to Cremanns-Otto [6] and Pride [22]. Given a monoid presentation $\mathcal{P}$ with generating set $A$, Squier associates to $\mathcal{P}$ a graph $\Gamma$ that has vertex set $A^{*}$ (the free monoid on $A$ ) and, for all $p, q \in A^{*}$, has an edge from $p u q$ to $p v q$ whenever $u=v$ is a relation in $\mathcal{P}$. A path in $\Gamma$ therefore corresponds to a chain of equivalences betwen words in $A^{*}$ as consequences of the relations in $\mathcal{P}$, and a homotopy relation is imposed to identify paths corresponding to such equivalences that are naturally considered to be essentially the same. If this homotopy relation is finitely generated, then

E-mail address: N.D.Gilbert@hw.ac.uk (corresponding author).

2010 Mathematics Subject Classification. Primary: 20M18, Secondary: 20L05, 20M50.

Key words and phrases. inverse monoid, presentation, groupoid, crossed module. 
$\mathcal{P}$ is said to have finite derivation type. For monoid presentations of groups, a theorem of Squier [26. Theorem 4.3] shows that if one finite presentation of $G$ has finite derivation type then all finite presentations of $G$ do. The main result of [6] is that finite derivation type (for groups) is equivalent to the homological finiteness property $\mathrm{FP}_{3}$.

An important component of the treatment of groups (given by monoid presentations) in [6] is the way in which free reductions are handled within the formalism. An approach based on the categorical algebra of monoidal groupoids and crossed modules, and refashioning the results of [22], was given in [9]. This approach is refined and extended in [11]. In any similar approach to presentations of inverse monoids, we encounter the problem of handling the Wagner congruence (see [27], for example), which defines the free inverse monoid as a quotient of a free monoid, and as mentioned above, is not finitely generated. To get around this problem, given a presentation $\mathcal{P}=[X: \mathcal{R}]$ of an inverse monoid $M$, we define a 2-complex as in [14, 21] whose edges encode the applications of relations, and whose 2-cells impose an appropriate homotopy relation, but we take as vertex set an inverse monoid $\mathcal{T}$ constructed canonically from $\mathcal{P}$. The presentation map $\operatorname{FIM}(X) \rightarrow M$ from the free inverse monoid on $X$ to $M$ factors through $\mathcal{T}$, which has $M$ as an idempotent separating image. We then work with the fundamental groupoid of this 2-complex: the use of groupoids in this general setting originates with the work of Kilibarda [14]. As a groupoid whose set of identities is an inverse monoid, our fundamental groupoid is an example of a pseudoregular groupoid, whose properties are considered in section 2 . We then aim to connect the structure of the fundamental groupoid with the relation module of $\mathcal{P}$ : for group presentations the $\mathrm{FP}_{3}$ condition is equivalent to finite presentation of the relation module.

We define the relation module of $\mathcal{P}$ in section 3 . We take a more direct approach than in earlier work of the first author [10], since the relation module can now be naturally defined in terms of the map $\mathcal{T} \rightarrow M$, and as in [10] we show that the relation module is isomorphic to the first homology of the Schützenberger graph of $(M, X)$. In section 4 we establish the connection between the relation module and the fundamental groupoid of our 2-complex. We use an intermediate construction of a free crossed module of groupoids, and derive a free presentation of the relation module as an $M$-module.

\section{BACKGROUND NOTIONS AND NOTATION}

Our basic reference for the theory of inverse semigroups is Lawson's book [16]. Aspects of the theories of groups and inverse semigroups are considered by side-by-side in [19]. We shall also make use of other algebraic constructions that may be less familiar, and we give brief introductions here.

1.1. Groupoids. A groupoid $G$ is a small category in which every morphism is invertible. We consider a groupoid as an algebraic structure (as in [12, 16]) whose elements are its morphisms, with a partial associative partial binary operation given by composition of morphisms. The set of vertices of $G$ is denoted $V(G)$, and for each vertex $x \in V(G)$ there exists an identity morphism $1_{x}$. An element $g \in G$ has domain $g \mathbf{d}$ and range $g \mathbf{r}$ in $V(G)$, with $g g^{-1}=1_{g \mathbf{d}}$ and $g^{-1} g=1_{g \mathbf{r}}$. For $e \in V(G)$ the star of $e$ in $G$ is the set $\operatorname{star}_{e}(G)=$ $\{g \in G: g \mathbf{d}=e\}$, and the local group at $e$ is the set $G(e)=\{g \in G: g \mathbf{d}=e=g \mathbf{r}\}$. 
Example 1.1. Let $X$ be any set and let $\rho \subseteq X \times X$ be an equivalence relation on $X$. Then $\rho$ is a groupoid with vertex set $X$, and with partial composition $(a, b)(c, d)=(a, d)$ if $b=c$. If $\rho=X \times X$ we obtain the simplicial groupoid on $X$.

Example 1.2. Let $X$ be a topological space and $A$ a subspace of $X$. Then the set of fixed-end-point homotopy classes of paths in $X$ with end-points in $A$ is a groupoid, the fundamental groupoid $\pi(X, A)$. We shall make use of the fundamental groupoid $\pi(X, A)$ of a 2-complex $X$, with $A$ its 0 -skeleton, in section 4 .

Example 1.3. An inverse semigroup $S$ may be considered as a groupoid $\vec{S}$, with $V(\vec{S})$ equal to the set of idempotents $E(S)$ of $S$. The groupoid composition $\circ$ on $\vec{S}$ is the restricted product on $S$ : the composition $s \circ t$ is defined if and only if $s^{-1} s=t t^{-1}$, and then $s \circ t=s t \in S$. This point of view is an important theme in [16].

1.2. Clifford Semigroups. Clifford semigroups constitute a class of inverse semigroups that will be of importance in the description of relation modules in section 3 .

Let $(E, \leqslant)$ be a meet semilattice, and let $\left\{G_{e}: e \in E\right\}$ be a family of groups indexed by the elements of $E$. For each pair $e, f \in E$ with $e \geqslant f$, let $\phi_{f}^{e}: G_{e} \rightarrow G_{f}$ be a group homomorphism, and suppose that the following two axioms hold:

- $\phi_{e}^{e}$ is the identity homomorphism on $G_{e}$,

- if $e \geqslant f \geqslant g$ then $\phi_{f}^{e} \phi_{g}^{f}=\phi_{g}^{e}$.

The collection

$$
\left(G_{e}, \phi_{f}^{e}\right)=\left(\left\{G_{e}: e \in E\right\},\left\{\phi_{f}^{e}: e, f \in E, f \leqslant e\right\}\right)
$$

is a presheaf of groups over $E$ and the group operations on the $G_{e}$ make the disjoint union $G=\bigsqcup_{e \in E} G_{e}$ into an inverse semigroup, called a Clifford semigroup over $E$, with binary operation

$$
x * y=\left(x \phi_{e f}^{e}\right)\left(y \phi_{e f}^{f}\right) \in G_{e f},
$$

where $x \in G_{e}$ and $y \in G_{f}$.

Our description of relation modules in section 3 also depends on the factorization of an inverse semigroup homomorphism from a free inverse monoid as a composition of an idempotent pure map and an idempotent separating map. We recall the definitions of these types of map here:

\section{Definition 1.1.}

(a) A congruence $\rho$ on an inverse semigroup $T$ is said to be idempotent pure if $a \in T$ and $a \rho e$ for some $e \in E(T)$ imply that $a \in E(T)$.

(b) A congruence $\rho$ on an inverse semigroup $T$ is said to be idempotent separating if $e, f \in E(T)$ and $e \rho f$ imply that $e=f$.

Any inverse semigroup homomorphism $\phi: T \rightarrow S$ induces a congruence $\chi_{\phi}$ on $T$ by

$$
a \chi_{\phi} b \Longleftrightarrow a \phi=b \phi \text {. }
$$

We say that $\phi$ is idempotent pure (respectively, idempotent separating) if $\chi_{\phi}$ has this property. The kernel of an inverse semigroup homomorphism $\phi: T \rightarrow S$ is the preimage of $E(S)$ :

$$
\operatorname{ker} \phi=\{a \in T: a \phi \in E(S)\} \text {. }
$$


We recall that any inverse semigroup $T$ has a maximum group image $\widehat{T}$ and that $T$ is $E$ unitary if the quotient map $\sigma_{T}: T \rightarrow \widehat{T}$ is idempotent pure. Free inverse monoids are $E$-unitary. See [16, section 2.4] for more properties of $E$-unitary inverse semigroups.

The connection that we need between Clifford semigroups and idempotent separating maps is given by the following result (see [16, Lemma 5.2.2]).

Proposition 1.4. If a homomorphism $\phi: T \rightarrow S$ of inverse semigroups is idempotent separating then its kernel is a Clifford semigroup over $E(T)$.

1.3. Schützenberger graphs. We shall use left Schützenberger graphs in this paper. Let $S$ be an inverse semigroup generated by a set $X$. There exists a presentation map $\theta$ : $\operatorname{FIS}(X) \rightarrow S$ from the free inverse semigroup on $X$ to $S$. The (left) Schützenberger graph $\operatorname{Sch}^{\mathcal{L}}(S, X)$ has vertex set $S$, and for $x \in X$ and $s \in S$, an $x$-labelled edge from $s$ to $(x \theta) s$ whenever $\left(x^{-1} x\right) \theta \geqslant s s^{-1}$. The connected component $\operatorname{Sch}^{\mathcal{L}}(S, X, e)$ containing the idempotent $e$ is the full subgraph on the vertex set $L_{e}$, the $\mathcal{L}$-class of $e$ in $S$. Some examples of Schützenberger graphs may be found in section 3.1

1.4. Modules for inverse semigroups. Modules for inverse semigroups were first defined by Lausch [15].

Definition 1.2. Let $S$ be an inverse semigroup with semilattice of idempotents $E(S)$. Consider a Clifford semigroup $\mathcal{A}=\left(A_{e}, \alpha_{f}^{e}\right)$ (see section 1.2), in which each $A_{e}$ is an additively written abelian group with identity $0_{e}$. The disjoint union $A=\bigsqcup_{e \in E(S)} A_{e}$ is a commutative inverse semigroup under the operation

$$
a \oplus b=a \alpha_{e f}^{e}+b \alpha_{e f}^{f}
$$

for $a \in A_{e}$ and $b \in A_{f}$. Then $\mathcal{A}$ is an $S$-module [15, section 2] if there exists a map $A \times S \rightarrow A$, written $(a, s) \mapsto a \triangleleft s$, such that

(i) $(a \oplus b) \triangleleft s=(a \triangleleft s) \oplus(b \triangleleft s)$ for all $a, b \in A$ and $s \in S$,

(ii) $a \triangleleft s t=(a \triangleleft s) \triangleleft t$ for all $a \in A$ and $s, t \in S$,

(iii) $a \triangleleft e=a \oplus 0_{e}$ for all $a \in A$ and $e \in E(S)$,

(iv) $0_{e} \triangleleft s=0_{s^{-1} e s}$ for all $e \in E(S)$ and $s \in S$.

A free $S$-module $\mathcal{F}=\left(F_{e}, \phi_{f}^{e}\right)$ has as basis a family of sets $\mathcal{B}=\left\{B_{e}: e \in E(S)\right\}$, and $F_{e}$ is the free abelian group on the set

$$
\left\{(b, s): b \in B_{f}, s \in S, f \geqslant s s^{-1}, s^{-1} s=e\right\},
$$

with $(b, s) \phi_{e^{\prime}}^{e}=\left(b, s e^{\prime}\right)$ and with $S$-action defined by $(b, s) \triangleleft t=(b, s t)$, see [15], section $3]$.

Lemma 1.5. Let $\psi: T \rightarrow S$ be a surjective idempotent separating homomorphism with kernel $K$. Then $\mathcal{K}=\bigsqcup_{e \in E(T)} K_{e}^{a b}$ is an $S$-module, with $S$-action defined by $k \triangleleft s=$ $t^{-1} k t$ for any $t \in T$ with $s=t \psi$.

1.5. Crossed modules of groupoids. We now present the rudiments of the theory of crossed modules of groupoids. For further information we refer to [5], and for the use of crossed modules in the theory of group presentations to [2].

Definition 1.3. Let $G$ be a groupoid with vertex set $V=V(G)$. Then a crossed $G$-module

$$
C \stackrel{\partial}{\rightarrow} G \rightrightarrows V
$$


consists of:

(1) a disjoint union of groups $C=\bigsqcup_{e \in V} C_{e}$, indexed by $V$,

(2) a homomorphism $\partial$ of groupoids,

(3) an action of $G$ on $C$, denoted $(c, g) \mapsto c^{g}$, such that an edge $g \in G$ with $g \mathbf{d}=e$ and $g \mathbf{r}=f$, acts on $c \in C_{e}$ with $c^{g} \in C_{f}$.

The action of $G$ on $C$ satisfies

$$
\begin{aligned}
& \left(c^{g}\right) \partial=g^{-1}(c \partial) g \quad \text { whenever } c^{g} \text { is defined, } \\
& c^{a \partial}=a^{-1} c a \quad \text { where, for some } e \in V, a, c \in C_{e} .
\end{aligned}
$$

Definition 1.4. Consider a crossed $G$-module

$$
C \stackrel{\partial}{\rightarrow} G \rightrightarrows V
$$

along with a set $R$ and a function $\omega: R \rightarrow G$ such that $\omega \mathbf{d}=\omega \mathbf{r}$. Then $C$ is said to be the free crossed $G$-module on $\omega$ if for any crossed $G$-module

$$
C^{\prime} \stackrel{\partial^{\prime}}{\rightarrow} G \rightrightarrows V
$$

and function $\sigma: R \rightarrow C^{\prime}$ such that $\omega=\sigma \partial^{\prime}$ there exists a unique morphism of crossed $G$-modules $\phi: C \rightarrow C^{\prime}$ such that $\partial=\phi \partial^{\prime}$.

We sketch the construction of free crossed modules: see [5, section 7.3].

Proposition 1.6. [5, Proposition 7.3.7] Given a groupoid $G$, a set $R$ and a function $\omega$ : $R \rightarrow G$ such that $\omega \mathbf{d}=\omega \mathbf{r}$, then a free crossed $G$-module on $\omega$ exists and is unique up to isomorphism.

Proof. For each $e \in V(G)$ we define $R_{e}=\{s \in R:(s \omega) \mathbf{r}=e=(s \omega) \mathbf{d}\}$ and

$$
(R \gamma G)_{e}=\left\{(s, g) \in R \times G: r \in R_{g g^{-1}}, g^{-1} g=e\right\} .
$$

We define $F_{e}$ to be the free group on $(R \varnothing G)_{e}$, and $\mathcal{F}=\bigsqcup_{e \in V} F_{e}$. Then we have a map $\delta: \mathcal{F} \rightarrow G$, defined on generators by $(s, g) \mapsto g^{-1}(s \omega) g$, and an action of $G$ on $\mathcal{F}$, defined on generators by $(s, g)^{h}=(s, g h)$ whenever $g^{-1} g=h h^{-1}$. We let $P_{e}$ denote the subgroup of $F_{e}$ generated by the elements of the form $\langle u, v\rangle=u^{-1} v^{-1} u v^{u \delta}$, for $u, v \in F_{e}$. Then $P_{e}$ is normal in $F_{e}$, invariant under the $G$-action, and contained in the kernel of $\delta$. So $\delta$ induces $\partial: \bigsqcup_{e \in V} F_{e} / P_{e} \rightarrow G$ and this is a free crossed module on $\omega$. Uniqueness follows from the usual universal argument.

We note that there exists a function $\nu: R \rightarrow C$ induced by mapping $s \in R_{e}$ to $(s, e) \in$ $(R \varnothing G)_{e}$, and that $\nu \partial=\omega$.

\subsubsection{Modules and crossed modules.}

Definition 1.5. Consider a crossed module $C \stackrel{\partial}{\rightarrow} G \rightrightarrows V$ in which $\partial$ is trivial: that is, $\partial$ maps each $a \in C_{e}$ to $e \in V$. We write $\partial=\varepsilon$. By CM2 each $C_{e}$ is then abelian, and $C$ is a $G$-module. The concept of a free $G$-module then follows: given a set $R$ and a function $\omega: R \rightarrow G$ with $\omega \mathbf{d}=\omega \mathbf{r}$, a $G$-module $\mathcal{A}$ is free on $\omega$, if for any $G$-module $\mathcal{B}$ and function $\nu: R \rightarrow \mathcal{B}$ such that $\nu \varepsilon=\omega$, there exists a unique morphism $\phi: \mathcal{A} \rightarrow \mathcal{B}$ of $G$-modules.

More generally, we have: 


\section{Proposition 1.7.}

(1) Let $\mathcal{C} \stackrel{\partial}{\rightarrow} G \rightrightarrows V$ be a crossed $G$-module, and let $\mathcal{Q}$ be the quotient groupoid $G / \mathcal{C} \partial$, with $\pi: G \rightarrow \mathcal{Q}$ the natural map. Then $\mathcal{C}^{a b}=\bigsqcup_{e \in V} C_{e}^{a b}$ is a $\mathcal{Q}$-module, where for $c \in C_{e}$ and $q=g \pi$ with $g g^{-1}=e$ we have

$$
\bar{c} \triangleleft q=\overline{c^{g}} .
$$

(2) If $\mathcal{C} \stackrel{\partial}{\rightarrow} G \rightrightarrows V$ is a free crossed module with basis $\omega: R \rightarrow G$ then $\mathcal{C}^{a b}$ is a free $\mathcal{Q}$-module with basis the image of the induced map $R \rightarrow \mathcal{C} \rightarrow \overline{\mathcal{C}}$.

Proof. The claimed $\mathcal{Q}$-action is well-defined, since if $q=g \pi=h \pi$ with $g, h \in G$, then $h=(a \partial) g$ for some $a \in \mathcal{C}$, and then by CM2,

$$
\overline{c^{h}}=\overline{c^{(a \partial) g}}=\overline{\left(a^{-1} c a\right)^{g}}=\overline{c^{g}} .
$$

Now let $\mathcal{A}$ be an arbitrary $\mathcal{Q}$-module, and consider the disjoint union of groups $\Lambda=$ $\sqcup_{e \in V} \Lambda_{e}$, where $\Lambda_{e}=C_{e} \partial \times A_{e}$. We let $G$ act on $\Lambda$ by conjugation on each $C_{e}$ and via $\pi$ on $A_{e}$. Let $p_{1}: \Lambda \rightarrow G$ be the projection map: we claim that $\Lambda \stackrel{p_{1}}{\longrightarrow} G \rightrightarrows V$ is a crossed $G$-module. For $(c \partial, a) \in \Lambda_{e}$ and $g \in G$ with $g \mathbf{d}=e$ we have:

$$
\left((c \partial, a)^{g}\right) p_{1}=\left(g^{-1}(c \partial) g, a^{g \pi}\right) p_{1}=g^{-1}(c \partial) g=g^{-1}(c \partial, a) p_{1} g,
$$

and for $\left(c_{1} \partial, a_{1}\right),\left(c_{2} \partial, a_{2}\right) \in \Lambda_{e}$,

$$
\begin{aligned}
\left(c_{1} \partial, a_{1}\right)^{\left(c_{2} \partial, a_{2}\right) p_{1}} & =\left(c_{1} \partial, a_{1}\right)^{c_{2} \partial} \\
& =\left(\left(c_{2} \partial\right)^{-1}\left(c_{1} \partial\right)\left(c_{2} \partial\right), a_{1}^{\left(c_{2} \partial\right) \pi}\right) \\
& =\left(\left(c_{2} \partial\right)^{-1}\left(c_{1} \partial\right)\left(c_{2} \partial\right), a_{1}\right)
\end{aligned}
$$

since $c_{2} \partial \pi=e$, and

$$
=\left(c_{2} \partial, a_{2}\right)^{-1}\left(c_{1} \partial, a_{1}\right)\left(c_{2} \partial, a_{2}\right),
$$

since $A_{e}$ is abelian. So $\Lambda \stackrel{p_{1}}{\longrightarrow} G \rightrightarrows V$ is a crossed $G$-module.

Now given $\nu^{\prime}: R \rightarrow \mathcal{A}$, we define

$$
\nu^{\prime \prime}=\left(\nu \partial, \nu^{\prime}\right): \mathcal{R} \rightarrow \Lambda .
$$

We note that $\nu^{\prime \prime} p_{1}=\nu \partial$, and so by freeness of $\mathcal{C}$, there is an induced morphism $\lambda: \mathcal{C} \rightarrow \Lambda$ of crossed $G$-modules, with $\nu \lambda=\nu^{\prime \prime}$. Composing $\lambda$ with the second projection $p_{2}: \Lambda \rightarrow$ $\mathcal{A}$ gives a morphism $\mathcal{C} \rightarrow \mathcal{A}$ that factors through $\mathcal{C}^{a b} \rightarrow \mathcal{A}$, and is easily seen to be a map of $\mathcal{Q}$-modules.

The maps used in the proof are illustrated below. 


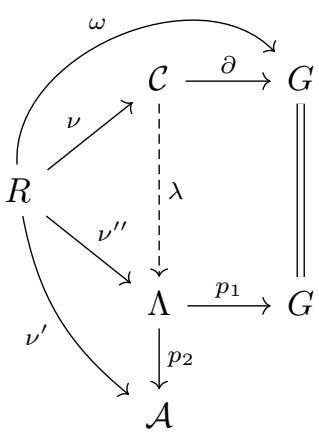

\section{SEMIREgUlAR AND PSEUDOREgUlAR GROUPOIDS}

We now introduce some additional structure on a groupoid, originating in work of Brown and Gilbert [3], and further developed by Gilbert in [9] and by Brown in [4].

Definition 2.1. Let $G$ be a groupoid, with object set $V(G)$ and domain and range maps $\mathbf{d}, \mathbf{r}: G \rightarrow V(G)$. Then $G$ is semiregular if

- $V(G)$ is a monoid, with identity $e \in V(G)$,

- there are left and right actions of $V(G)$ on $G$, denoted $x \triangleright \alpha, \alpha \triangleleft x$, which for all $x, y \in V(G)$ and $\alpha, \beta \in G$ satisfy:

(a) $(x y) \triangleright \alpha=x \triangleright(y \triangleright \alpha) ; \alpha \triangleleft(x y)=(\alpha \triangleleft x) \triangleleft y$; $(x \triangleright \alpha) \triangleleft y=x \triangleright(\alpha \triangleleft y)$,

(b) $e \triangleright \alpha=\alpha=\alpha \triangleleft e$,

(c) $(x \triangleright \alpha) \mathbf{d}=x(\alpha \mathbf{d}) ;(\alpha \triangleleft x) \mathbf{d}=(\alpha \mathbf{d}) x ;(x \triangleright \alpha) \mathbf{r}=x(\alpha \mathbf{r}) ;(\alpha \triangleleft x) \mathbf{r}=(\alpha \mathbf{r}) x$,

(d) $x \triangleright(\alpha \circ \beta)=(x \triangleright \alpha) \circ(x \triangleright \beta) ;(\alpha \circ \beta) \triangleleft x=(\alpha \triangleleft x) \circ(\beta \triangleleft x)$, whenever $\alpha \circ \beta$ is defined,

(e) $x \triangleright 1_{y}=1_{x y}=1_{x} \triangleleft y$.

From [9, section 1] we have the following facts.

\section{Proposition 2.1.}

(a) Let $G$ be a semiregular groupoid. Then there are two everywhere defined binary operations on $G$ given by:

$$
\begin{aligned}
\alpha * \beta & =(\alpha \triangleleft \beta \mathbf{d}) \circ(\alpha \mathbf{r} \triangleright \beta) \\
\alpha \circledast \beta & =(\alpha \mathbf{d} \triangleright \beta) \circ(\alpha \triangleleft \beta \mathbf{r}) .
\end{aligned}
$$

Each of the binary operations $*$ and $\circledast$ make $G$ into a monoid, with identity $1_{e}$.

(b) The binary operation $*$ and the monoid structure on $V(G)$ make the semiregular groupoid $G$ into a strict monoidal groupoid if and only if the operations $*$ and $\circledast$ on $G$ coincide.

Definition 2.2. In view of part (c) of Proposition 2.1, we say that a semiregular groupoid is monoidal if the operations $*$ and $\circledast$ coincide. (Brown [4] calls such semiregular groupoids commutative whiskered groupoids.) 
2.1. Pseudoregular groupoids. In considering presentations of inverse monoids, we shall want to consider semiregular groupoids in which the vertex set is an inverse monoid.

Definition 2.3. A semiregular groupoid $G$ is a pseudoregular groupoid if $V(G)$ is an inverse monoid.

The name pseudoregular is chosen to reflect the close structural connection between inverse monoids and pseudogroups, which are inverse semigroups of partial homeomorphisms of topological spaces (see [16, section 1.1]).

In a pseudoregular groupoid $G$, the operations, $*$ and $\circledast$ given in proposition 2.1 a) each make $G$ into a monoid, but not necessarily an inverse monoid, as we show in the next example.

Example 2.2. Let $\partial: T \rightarrow G$ be a crossed module of groups. Add a zero 0 to $G$ to form the inverse semigroup $G^{0}$ and let $0 \in G^{0}$ act on $T$ as the trivial endomorphism $t \mapsto 1$. The product $G^{0} \times T$ is then a pseudoregular groupoid, with the following structure. The subset $G \times T$ is a semiregular groupoid (see [9. Proposition 1.3(ii)]) with vertex set $G$, with $(g, t) \mathbf{d}=g$ and $(g, t) \mathbf{r}=g(t \partial)$, and with composition $(g, t)(h, u)=(g, t u)$ defined when $h=h(t \partial)$. For the additional arrows in $\{0\} \times T$ we define $(0, t) \mathbf{d}=0=(0, t) \mathbf{r}$ and composition $(0, t) \circ(0, u)=(0, t u)$ and so the local group at 0 is a copy of $T$. The left and right actions of $0 \in G^{0}$ are given by:

$$
\begin{aligned}
& 0 \triangleright(g, t)=(0,1)(g, t)=(0, t), \\
& (g, t) \triangleleft 0=(g, t)(0,1)=(0,1), \\
& 0 \triangleright(0, t)=(0,1)(0, t)=(0, t), \\
& (0, t) \triangleleft 0=(0, t)(0,1)=(0,1) .
\end{aligned}
$$

Then $\partial: T \rightarrow G^{0}$ is a crossed monoid (originally monoide croisé) in the sense of Lavendhomme and Roisin [17, Example 1.3C], and $G^{0} \times T$ is a pseudoregular groupoid $G$, with vertex set $G^{0}$. The $*$-operation on $G$ recovers the semidirect product $G^{0} \ltimes T$ :

$$
(g, t) *(h, u)=\left(g h, t^{h} u\right)
$$

and the operations $*$ and $\circledast$ coincide, but $G^{0} \ltimes T$ is not inverse. This follows from the results of [20], but can also be seen directly, as follows.

For any $t \in T$, the element $(0, t)$ is an idempotent in $\left(G^{0} \ltimes T, *\right)$ :

$$
(0, t) *(0, t)=\left(0, t^{0} t\right)=(0,1 t)=(0, t) .
$$

But for distinct $s, t \in T$ we have

$$
(0, s) *(0, t)=\left(0, s^{0} t\right)=(0, t) \text { and }(0, t)(0, s)=(0, s)
$$

and so the idempotents in $G^{0} \ltimes T$ do not commute. Since $G \ltimes T$ is a subgroup of $G^{0} \ltimes T$ and the other elements are idempotents, $G^{0} \ltimes T$ is regular (and indeed orthodox, since $E\left(G^{0} \ltimes T\right)$ is a subsemigroup).

In a pseudoregular groupoid $G$, it is natural to consider $\operatorname{star}_{e}(G)$ for each idempotent $e \in V(G)$. The operation $*$ then $\operatorname{makes} \operatorname{star}_{e}(G)$ into a semigroup. However, as the following example shows, the identity arrow $1_{e}$ at $e$ is not necessarly an identity element for $\left(\operatorname{star}_{e}(G), *\right)$. 
Example 2.3. Let $E$ be the semilattice $\{1, e, f, 0\}$ with ef $=0$ and consider the simplicial groupoid $E \times E$ with vertex set $E$, and $\mathbf{d}$ and $\mathbf{r}$ given by the projection maps. Let $U$ be the subgroupoid of $E \times E$ defined by

$$
U=\{(x, y) \in E \times E: x \neq 1 \neq y\} \cup\{(1,1)\} .
$$

Right and left actions of $E$ on $E \times E$ are defined by multiplication:

$$
x \triangleright(y, z)=(x y, x z) \quad \text { and } \quad(x, y) \triangleleft z=(x z, y z),
$$

making $U$ pseudoregular. The $*$-operation is given by

$$
(u, v) *(x, y)=((u, v) \triangleleft x)(v \triangleright(x, y))=(u x, v x)(v x, v y)=(u x, v y) .
$$

The star at 0 is $\operatorname{star}_{0}=\{(0, e),(0, f),(0,0)\}$, but the identity arrow $1_{0}=(0,0)$ is not an identity element in $\left(\operatorname{star}_{0}, *\right)$.

We can, however, remedy the problem illustrated in Example 2.3 by passing to a subsemigroup that does admit $1_{e}$ as an identity. For an idempotent $e \in V(G)$ we define

$$
\operatorname{star}_{e}^{\bowtie}(G)=\left\{e \triangleright \alpha \triangleleft e: \alpha \in \operatorname{star}_{e}(G)\right\} .
$$

It is clear that the operation $*$ now makes $\operatorname{star}_{e}^{\bowtie}(G)$ into a monoid with identity $1_{e}$. The range map $\mathbf{r}: G \rightarrow V(G)$ restricts to a semigroup morphism $\mathbf{r}_{e}: \operatorname{star}_{e}^{\bowtie}(G) \rightarrow V(G)$ whose image is a monoid $K_{e}$ with identity $e$. For $\alpha \in \operatorname{star}_{e}(G)$ we set $\alpha^{\bowtie}=e \triangleright \alpha \triangleleft e$ and define

$$
\pi_{e}^{\bowtie}(G)=\left\{\alpha^{\bowtie} \in \operatorname{star}_{e}^{\bowtie}(G):\left(\alpha^{\bowtie}\right) \mathbf{r}=e\right\} .
$$

Proposition 2.4. In a pseudoregular groupoid $G$, the binary operation $*$ and the groupoid composition $\circ$ coincide on $\pi_{e}^{\bowtie}(G)$ and under each operation $\pi_{e}^{\bowtie}(G)$ is a group. Furthermore if $G$ is monoidal, then $\pi_{e}^{\bowtie}(G)$ is abelian, and the family of abelian groups $\pi^{\bowtie}(G)=\left\{\pi_{e}^{\bowtie}(G), e \in E(V(G))\right\}$, is a $V(G)$-module.

Proof. For $\alpha^{\bowtie}, \beta^{\bowtie} \in \pi_{e}^{\bowtie}(G)$ we have

$$
\alpha^{\bowtie} * \beta^{\bowtie}=\left(\alpha^{\bowtie} \triangleleft\left(\beta^{\bowtie}\right) \mathbf{d}\right) \circ\left(\left(\alpha^{\bowtie}\right) \mathbf{r} \triangleright \beta^{\bowtie}\right)=\left(\alpha^{\bowtie} \triangleleft e\right) \circ\left(e \triangleright \beta^{\bowtie}\right)=\alpha^{\bowtie} \circ \beta^{\bowtie} .
$$

Since $e \triangleright \alpha^{-1} \triangleleft e=(e \triangleright \alpha \triangleleft e)^{-1}$ it is clear that $\pi_{e}^{\bowtie}(G)$ is a subgroup of the local group $\pi_{1}(G, e)$ at $e$ in the groupoid $G$.

If $G$ is monoidal, then $*$ and $\circledast$ coincide, and

$$
\alpha^{\bowtie} \circ \beta^{\bowtie}=\alpha^{\bowtie} * \beta^{\bowtie}=\alpha^{\bowtie} \circledast \beta^{\bowtie}=\left(e \triangleright \beta^{\bowtie}\right) \circ\left(\alpha^{\bowtie} \triangleleft e\right)=\beta^{\bowtie} \circ \alpha^{\bowtie} .
$$

So $\pi_{e}^{\bowtie}$ is abelian. Now for $e \geqslant f$ we define $\varphi_{f}^{e}: \pi_{e}^{\bowtie}(G) \rightarrow \pi_{f}^{\bowtie}(G)$ by $\alpha^{\bowtie} \mapsto f \triangleright \alpha^{\bowtie} \triangleleft f \in$ $\pi_{f}^{\bowtie}$. Then for $\alpha^{\bowtie}, \beta^{\bowtie} \in \pi_{e}^{\bowtie}$ :

$$
\begin{aligned}
\left(\alpha^{\bowtie} * \beta^{\bowtie}\right) \varphi_{f}^{e} & =f \triangleright\left(\alpha^{\bowtie} * \beta^{\bowtie}\right) \triangleleft f \\
& =f \triangleright\left(\alpha^{\bowtie} \circ \beta^{\bowtie}\right) \triangleleft f \\
& =\left(f \triangleright \alpha^{\bowtie} \triangleleft f\right) \circ\left(f \triangleright \beta^{\bowtie} \triangleleft f\right) \\
& =\left(f \triangleright \alpha^{\bowtie} \triangleleft f\right) *\left(f \triangleright \beta^{\bowtie} \triangleleft f\right) \\
& =\alpha^{\bowtie} \varphi_{f}^{e} * \beta^{\bowtie} \varphi_{f}^{e}
\end{aligned}
$$

and so each $\varphi_{f}^{e}$ is a homomorphism. Furthermore, if $e \geqslant f \geqslant g$ then

$$
\alpha^{\bowtie} \varphi_{f}^{e} \varphi_{g}^{f}=g \triangleright\left(f \triangleright \alpha^{\bowtie} \triangleleft f\right) \triangleleft g=g f \triangleright \alpha^{\bowtie} \triangleleft f g=g \triangleright \alpha^{\bowtie} \triangleleft g=\alpha^{\bowtie} \varphi_{g}^{e}
$$


and clearly $\varphi_{e}^{e}$ is the identity on $\pi_{e}^{\bowtie}$. Therefore $\left(\pi_{e}^{\bowtie}, \varphi_{f}^{e}\right)$ is a presheaf of abelian groups and a $V(G)$-action is now given by $\alpha \triangleleft s=s^{-1} \triangleright \alpha \triangleleft s \in \pi_{s^{-1} e s}^{\bowtie}$. It is easy to check that the conditions in Definition 1.2 for a Lausch $V(G)$-module are satisfied.

\section{THE RELATION MODULE OF AN INVERSE MONOID PRESENTATION}

Let $G$ be a group generated by a set $X$, with corresponding presentation map $\theta: F(X) \rightarrow$ $G$. Let $N$ be the kernel of $\theta$ : then conjugation in $F(X)$ induces a $G$-action on the abelianisation $N^{a b}$ of $N$, and $N^{a b}$ is the relation module. As shown in [2, Corollary 5.1], the relation module is isomorphic to the first homology group of the Cayley graph $\operatorname{Cay}(G, X)$.

In [10] the first author introduced relation modules for inverse monoid presentations by adapting work of Crowell [7] on group presentations. It was remarked in [10] that

Defining the relation module in this way permits the introduction of the concept in other algebraic settings where the operation of abelianisation has no obvious counterpart.

However, it turns out (as we shall see below) that we can indeed define the relation module of an inverse monoid presentation as the abelianisation of a certain Clifford semigroup, in a precise analogy of the construction for groups. We first describe a factorization result for inverse semigroups homomorphisms. Our discussion is based on [18, page 265], to which we refer for further details. The result originates in [23, Theorem 4.2].

Proposition 3.1. Let $\rho$ be a congruence on the inverse semigroup $S$. Then there exists a smallest congruence $\rho_{\min }$ on $S$ whose trace is the same as the trace of $\rho$, defined by

$$
a \rho_{\min } b \Longleftrightarrow \text { there exists } e \in E(S) \text { with } a e=b e \text { and } a^{-1} a \rho \text { e } \rho b^{-1} b .
$$

Furthermore,

(a) For $a, b \in S$ we have

$$
a \rho_{\min } b \Longleftrightarrow \text { there exists } c \in S \text { with } a \geqslant c \leqslant b \text { and } a \rho c \rho b .
$$

(b) The canonical map $\psi: S / \rho_{\min } \rightarrow S / \rho$ is idempotent separating,

(c) If $S$ is E-unitary then the canonical map $\tau: S \rightarrow S / \rho_{\min }$ is idempotent pure.

Proof. (a) We first show that the conditions (3.1) and (3.2) are equivalent. First assume that (3.1) holds and set $c=a e=b e$. Then $a \geqslant c \leqslant b$ and, since $a^{-1} a \rho e \rho b^{-1} b$ we have

$$
a=a a^{-1} a \rho a e=b e \rho b b^{-1} b=b .
$$

Now if 3.2 holds, take $e=c^{-1} c$. Since $a \geqslant c \leqslant b$ we have $a e=c=b e$, and since $a \rho c$ we have $a^{-1} a \rho c^{-1} c=e$. Similarly $b^{-1} b \rho c$.

(b) Suppose that $a, b \in S$ with $a \rho_{\min } a^{2}$ and $b \rho_{\min } b^{2}$. By Lallement's Lemma [13, Lemma 2.4.3], there exist $e, f \in S$ with $a \rho_{\min } e$ and $b \rho_{\min } f$. If now $e \rho f$ then $e \rho_{\min } f$, and so $a \rho_{\min } b$. Hence $\psi$ is idempotent separating.

(c) Suppose that, for $s \in S$ and $x \in E(S)$, we have $s \rho_{\min } x$. Then there exists $e \in E(S)$ with $s e=x e$ and $x e \in E(S)$, and if $S$ is $E$-unitary, we have $s \in E(S)$ and so $\tau$ is idempotent pure. 
We now consider an inverse monoid presentation $\mathcal{P}=[X: \mathcal{R}]$ of an inverse monoid $M$. We set $A=X \sqcup X^{-1}$, and so $M$ is then a quotient of the free monoid $A^{*}$, with canonical $\operatorname{map} \varphi: A^{*} \rightarrow M$, and also a quotient of the free inverse monoid $\operatorname{FIM}(X)$, with associated presentation map $\theta: \operatorname{FIM}(X) \rightarrow M$. The Wagner congruence on $A^{*}$ induces the natural map $\rho: A^{*} \rightarrow \operatorname{FIM}(X)$, and $\varphi=\rho \theta$, and we may factorize $\theta$ as in Proposition 3.1 We set $\mathcal{T}(M, X)=\operatorname{FIM}(X) / \theta_{\min }$ and so have the commutative diagram

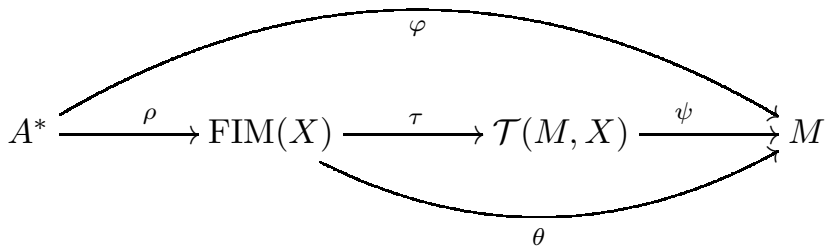

Since $\operatorname{FIM}(X)$ is $E$-unitary, the map $\tau$ is idempotent pure and we obtain from [18] the following structural information on $\mathcal{T}(M, X)$.

Lemma 3.2. [18, Lemma 1.6] Let $\mathcal{P}=[X: \mathcal{R}]$ be a presentation of an inverse monoid $T$. Then the following are equivalent:

(a) the presentation map $\theta: \operatorname{FIM}(X) \rightarrow T$ is idempotent pure.

(b) $\mathcal{P}$ is equivalent to a presentation of the form $\mathcal{P}_{1}=\left[X ; \mathcal{R}_{1}\right]$ where $\mathcal{R}_{1}=\left\{e_{i}=f_{i}\right.$ : $i \in I\}$ for some set I and idempotents $e_{i}, f_{i}$ of $\operatorname{FIM}(X)$.

(c) Each Schützenberger graph, $\mathrm{Sch}^{\mathcal{L}}(T, X, e)$ is a tree.

Definition 3.1. An inverse monoid $T$ is arboreal [10] if it satisfies the conditions of Lemma 3.2

Corollary 3.3. An arboreal inverse monoid $T$ is E-unitary.

Proof. It follows from part (b) of Lemma 3.2 that $T$ has maximum group image $F(X)$ and that the quotient map $\sigma: \operatorname{FIM}(X) \rightarrow F(X)$ factorizes as $\tau \sigma_{T}$. Since $\sigma$ is idempotent pure and $\tau$ is surjective, the map $\sigma_{T}$ is idempotent pure.

The factorization of $\theta$ shown in 3.3 gives us an idempotent separating homomorphism $\psi: \mathcal{T}(M, X) \rightarrow M$. By Proposition 1.4

$$
K=\operatorname{ker} \psi=\{w \in \mathcal{T}(M, X): w \psi \in E(M)\} .
$$

is a Clifford semigroup, and so is a union of groups $K_{e}$, indexed by the idempotents of $M$. Hence $K$ has the natural abelianisation

$$
\mathcal{K}=\bigcup_{e \in E(M)} K_{e}^{a b}
$$

that is an $M$-module by Lemma 1.5 .

Definition 3.2. The relation module of the presentation $\mathcal{P}$ is the $M$-module $\mathcal{K}$.

We now draw the connection between relation modules and Schützenberger graphs. For the left $\operatorname{Schützenberger~graph~} \operatorname{Sch}^{\mathcal{L}}(M, X)$, the cellular chain group $C_{0}\left(\operatorname{Sch}^{\mathcal{L}}(M, X, e)\right)$ is the free abelian group on the $\mathcal{L}$-class $L_{e}$ in $M$, and $C_{1}\left(\operatorname{Sch}^{\mathcal{L}}(M, X, e)\right)$ is the free abelian group on the set

$$
\left\{(x, s): x \in X, s \in M,\left(x^{-1} x\right) \theta \geqslant s s^{-1}, s^{-1} s=e\right\} .
$$


The boundary map $\partial: C_{1}\left(\operatorname{Sch}^{\mathcal{L}}(M, X, e)\right) \rightarrow C_{0}\left(\operatorname{Sch}^{\mathcal{L}}(M, X, e)\right)$ maps $(x, s) \mapsto(x \theta) s-$ $s$. Now

$$
C_{0}\left(\operatorname{Sch}^{\mathcal{L}}(M, X)\right)=\bigoplus_{e \in E(M)} C_{0}\left(\operatorname{Sch}^{\mathcal{L}}(M, X, e)\right)
$$

and

$$
C_{1}\left(\operatorname{Sch}^{\mathcal{L}}(M, X)\right)=\bigoplus_{e \in E(M)} C_{1}\left(\operatorname{Sch}^{\mathcal{L}}(M, X, e)\right)
$$

and by defining

$$
s \triangleleft t=s t \text { and }(a, s) \triangleleft t=(a, s t)
$$

we get an $M$-module structure on each of $C_{0}\left(\operatorname{Sch}^{\mathcal{L}}(M, X)\right)$ and $C_{1}\left(\operatorname{Sch}^{\mathcal{L}}(M, X)\right)$. The boundary map $\partial: C_{1}\left(\operatorname{Sch}^{\mathcal{L}}(M, X)\right) \rightarrow C_{0}\left(\operatorname{Sch}^{\mathcal{L}}(M, X)\right)$ is then a map of $M$-modules, and its kernel $H_{1}\left(\operatorname{Sch}^{\mathcal{L}}(M, X)\right)$ is an $M$-module $\mathcal{H}$, with the group $H_{1}\left(\operatorname{Sch}^{\mathcal{L}}(M, X)\right)_{e}$ being the first homology group $H_{1}\left(\operatorname{Sch}^{\mathcal{L}}(M, X, e)\right)$ of the connected component containing $e \in E(M)$.

For $e \in E(M)$ we define $U_{e}=\left\{w \in A^{*}: w \varphi \geqslant e\right\}$. Then $U_{e}$ is a submonoid of $A^{*}$ and is the reverse of the language accepted by the $\operatorname{Schützenberger}$ graph $\operatorname{Sch}^{\mathcal{L}}(M, X, e)$ when regarded as an automaton with input alphabet $A$ and unique start/accept state $e$, see [27]. (The reversal arises because we assume that as an automaton, $\operatorname{Sch}^{\mathcal{L}}(M, X, e)$ reads input words from the left, but the action on states is by left multiplication in $M$.) Let $\pi_{e}$ denote the fundamental group $\pi_{1}\left(\operatorname{Sch}^{\mathcal{L}}(M, X, e), e\right)$. A closed path $\alpha$ in $\operatorname{Sch}^{\mathcal{L}}(M, X, e)$ is labelled by a unique word $w \in A^{*}$ whose reverse $w^{\mathrm{R}}$ is in $U_{e}$ : we write $[w]$ for the homotopy class of $\alpha$ in $\pi_{e}$.

Lemma 3.4. There is a group isomorphism $\kappa_{e}: \pi_{e} \rightarrow K_{e}$ mapping the homotopy class $[w] \in \pi_{e}$ to $\left[\left(w^{\mathrm{R}} \rho \tau\right) \tilde{e}\right]^{-1}$, where $w^{\mathrm{R}}$ is the reverse of $w$ and $\tilde{e}$ is the unique preimage in $E(\mathcal{T}(M, X))$ of $e \in E(M)$.

Proof. If $[w] \in \pi_{e}$ then $w^{\mathrm{R}} \in U_{e}$ and

$$
\psi:\left(w^{\mathrm{R}} \rho \tau\right) \tilde{e} \mapsto\left(w^{\mathrm{R}} \rho \tau \psi\right)(\tilde{e} \psi)=\left(w^{\mathrm{R}} \varphi\right) e=e .
$$

Hence $\left[\left(w^{\mathrm{R}} \rho \tau\right) \tilde{e}\right]^{-1} \in K_{e}$. To verify that $\kappa_{e}$ is well-defined on $\pi_{e}$, suppose that $[u]=[v]$. Then $v$ can be obtained from $u$ by the insertion and deletion of subwords $a a^{-1}$ with $a \in A$. Considering one such step, if for some $p, q \in A^{*}$ we have $u=p q$ and $v=p a a^{-1} q$ then

$$
\left[\left(u^{\mathrm{R}} \rho \tau\right) \tilde{e}\right]^{-1} \geqslant\left[\left(v^{\mathrm{R}} \rho \tau\right) \tilde{e}\right]^{-1}
$$

in the subgroup $K_{e}$ of $\mathcal{T}(M, X)$ : since the relation $\geqslant$ is trivial on $K_{e}$, we deduce that $\left[\left(u^{\mathrm{R}} \rho \tau\right) \tilde{e}\right]^{-1}=\left[\left(v^{\mathrm{R}} \rho \tau\right) \tilde{e}\right]^{-1}$, and so $\kappa_{e}$ is well-defined.

Now for $u, v \in U_{e}$ we have

$$
\begin{aligned}
{[u] \cdot[v]=[u v] \mapsto\left[\left((u v)^{\mathrm{R}} \rho \tau\right) \tilde{e}\right]^{-1} } & =\tilde{e}\left(u^{\mathrm{R}} \rho \tau\right)^{-1}\left(v^{\mathrm{R}} \rho \tau\right)^{-1} \\
& =\tilde{e}\left(u^{\mathrm{R}} \rho \tau\right)^{-1} \tilde{e}\left(v^{\mathrm{R}} \rho \tau\right)^{-1}=\left([u] \kappa_{e}\right)\left([v] \kappa_{e}\right),
\end{aligned}
$$

since $\tilde{e}$ is the identity of the group $K_{e}$ and $\tilde{e}\left(u^{\mathrm{R}} \rho \tau\right)^{-1} \in K_{e}$. Hence $\kappa_{e}$ is a homomorphism.

If $k \in K_{e}$ we set $w_{k}$ to be any word in $A^{*}$ with $w_{k}^{\mathrm{R}} \rho \tau=k$. Then $e=w_{k}^{\mathrm{R}} \rho \tau \psi=w_{k}^{\mathrm{R}} \varphi$ and so $w_{k}^{\mathrm{R}} \in U_{e}$ and $\left[w_{k}\right] \in \pi_{e}$. Therefore $\kappa_{e}$ is surjective. 
Now suppose that $[w] \in \operatorname{ker} \kappa_{e}$. Then $\left(w^{\mathrm{R}} \rho \tau\right) \tilde{e}=\tilde{e} \in \mathcal{T}(M, X)$, and since $\mathcal{T}(M, X)$ is $E$-unitary, we deduce that $w^{\mathrm{R}} \rho \tau \in E(\mathcal{T})$. Since $\tau$ is idempotent pure, $w^{\mathrm{R}} \rho \in E(\operatorname{FIM}(X))$ and $w^{\mathrm{R}}$ is freely reducible to the empty word: hence the circuit at $e$ in $\operatorname{Sch}^{\mathcal{L}}(M, X, e)$ labelled by $w$ is homotopic to the constant path at $e$, and $[w]$ is trivial. Therefore $\kappa_{e}$ is injective.

Theorem 3.5. The $M$-modules

$$
\mathcal{K}=\bigsqcup_{e \in E(M)} K_{e}^{a b} \quad \text { and } \quad \mathcal{H}=\bigsqcup_{e \in E(M)} H_{1}\left(\operatorname{Sch}^{\mathcal{L}}(M, X, e)\right)
$$

are isomorphic.

Proof. We identify $H_{1}\left(\operatorname{Sch}^{\mathcal{L}}(M, X, e)\right)$ with $\pi_{e}^{a b}$ to exploit Lemma 3.4 for each $e \in$ $E(M)$ there is a group isomorphism $\bar{\kappa}_{e}: \pi_{e}^{a b} \rightarrow K_{e}^{a b}$. The action of $t \in M$ on $\mathcal{H}$ is induced by the family of maps $\pi_{e}^{a b} \rightarrow \pi_{t^{-1} e t}^{a b}$, in which the image of a closed path $[w]$ in $\pi_{e}^{a b}$ is mapped to the image of $\left[u^{-1} w u\right]$ in $\pi_{t^{-1} e t}^{a b}$, where $t^{-1}=u^{\mathrm{R}} \varphi$ (and so $u$ labels a path from et to $t^{-1} e t$ in $\left.\operatorname{Sch}^{\mathcal{L}}\left(M, X, t^{-1} e t\right)\right)$. We note that the isomorphism $\kappa_{t^{-1}} e t$ maps

$$
\left[u^{-1} w u\right] \mapsto\left(u^{-1} w u\right)^{\mathrm{R}} \rho \tau \cdot \widetilde{t^{-1} e t},
$$

where $\widetilde{t^{-1} e t}$ is the unique element of $E(\mathcal{T}(M, X))$ with $\left(\widetilde{t^{-1} e t}\right) \psi=t^{-1}$ et.

By Lemma 1.5, the $M$-action on $\mathcal{K}$ is induced by conjugation in $\mathcal{T}(M, X)$ : for $k \in K_{e}$ with image $\bar{k} \in K_{e}^{a b}$,

$$
\bar{k} \triangleleft t=\overline{\tilde{t}^{-1} k \tilde{t}} \in K_{t^{-1}}^{a b} e t
$$

for any $\tilde{t}$ with $\tilde{t} \psi=t$. We set $\tilde{t}=\left(u^{-1}\right)^{\mathrm{R}} \rho \tau$. Then for $[w] \in \pi_{e}$,

$$
\begin{aligned}
\tilde{t}^{-1}\left([w] \kappa_{e}\right) \tilde{t} & =\tilde{t}^{-1}\left(w^{\mathrm{R}} \rho \tau\right) \tilde{e} \tilde{t} \\
& =\tilde{t}^{-1}\left(w^{\mathrm{R}} \rho \tau\right) \tilde{t} \tilde{t}^{-1} \tilde{e} \tilde{t} \\
& =\left(u^{\mathrm{R}} \rho \tau\right)\left(w^{\mathrm{R}} \rho \tau\right)\left(\left(u^{-1}\right)^{\mathrm{R}} \rho \tau\right) \tilde{t}^{-1} \tilde{e} \tilde{t} \\
& =\left(\left(u^{-1} w u\right)^{\mathrm{R}} \rho \tau\right) \cdot \widetilde{t^{-1} e t}
\end{aligned}
$$

using the fact that $\psi$ is idempotent separating. Therefore the diagram

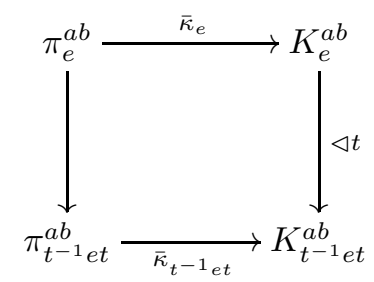

commutes, and the family of maps $\left\{\bar{\kappa}_{e}: E \in E(M)\right\}$ is an $M$-module isomorphism.

\subsection{Examples of relation modules.}


Example 3.6. Let $M$ be the semilattice $\{1, e, f, e f\}$, generated as an inverse monoid by $\{e, f\}$. The Schützenberger graph is

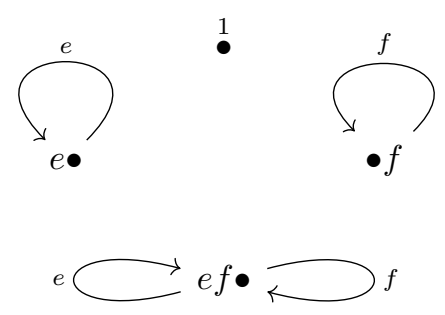

and the relation module is therefore

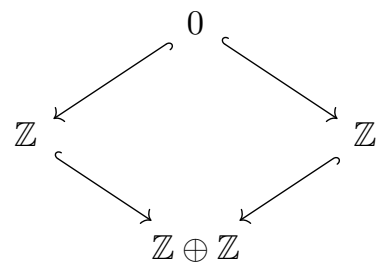

where all the structure maps are inclusions.

Example 3.7. The bicyclic monoid $B$ is the inverse monoid presented by $\left[x: x x^{-1}=1\right]$. The Schützenberger graph $\operatorname{Sch}^{\mathcal{L}}\left(B, x, x^{-q} x^{q}\right)$ is the semi-infinite path

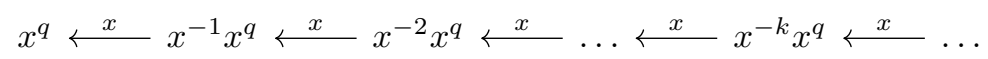

The relation module $\mathcal{K}$ is therefore trivial. This is no surprise: $B$ is an arboreal inverse monoid, and this Example illustrates Lemma 3.2

Example 3.8. Given an inverse monoid $M$ with presentation $[Y: R]$, we add a zero to $M$ to obtain $M^{0}$. For $M^{0}$ we take the generating set $X=Y \cup\{z\}$ (with $z \notin Y$ ), and we have a presentation $\mathcal{Q}$ of $M^{0}$ given by

$$
\mathcal{Q}=\left[Y, z: R, z^{2}=z, y z=z=z y(y \in Y)\right] .
$$

In the Schützenberger graph there is a loop at 0 for each element of $X$. If $[Y: R]$ has relation module $\mathcal{K}$ then the relation module of $\mathcal{Q}$ can be thought of schematically as

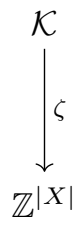

where the map $\zeta$ carries a circuit in $\operatorname{Sch}^{\mathcal{L}}(M, Y)$ labelled by a word $w \in\left(Y \sqcup Y^{-1}\right)^{*}$ to the element of $\mathbb{Z}^{|Y|} \subset \mathbb{Z}^{|X|}$ determined by $w$.

Example 3.9. The symmetric inverse monoid $\mathcal{I}_{2}$ on the set $\{1,2\}$ is generated by the transposition $\tau$ and the identity map $\varepsilon$ on $\{1\}$ : then $\varepsilon \tau \varepsilon$ is the empty map $\mathbf{0}$, and $\mathcal{I}_{2}=$ 
$\{\mathbf{1}, \tau, \varepsilon, \tau \varepsilon, \varepsilon \tau, \tau \varepsilon \tau, \mathbf{0}\}$. The Schützenberger graph $\operatorname{Sch}^{\mathcal{L}}\left(\mathcal{I}_{2},\{\tau, \varepsilon\}\right)$ is

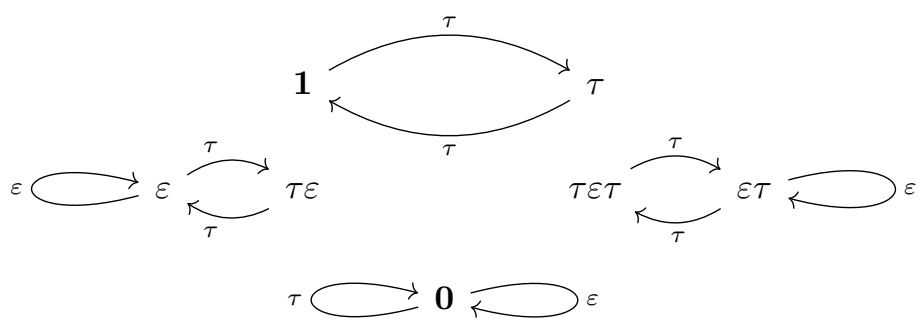

The relation module is therefore

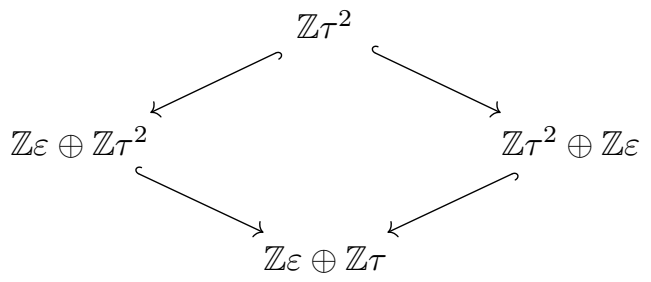

\section{THE SQUIER COMPLEX OF AN INVERSE MONOID PRESENTATION}

In this section we show that we can obtain a presentation of the relation module $\mathcal{K}$, derived from an inverse monoid presentation $\mathcal{P}=[X: \mathcal{R}]$ with presentation map $\theta: \operatorname{FIM}(X) \rightarrow$ $M$, from a free crossed module that is in turn derived from a $\operatorname{Squier} \operatorname{complex} \operatorname{Sq}(\mathcal{P})$ associated to $\mathcal{P}$.

Definition 4.1. Let $\mathcal{P}=[X: \mathcal{R}]$ be an inverse monoid presentation of $M$, with presentation map $\theta: \operatorname{FIM}(X) \rightarrow M$ factorised as in (3.3), as

$$
F I M(X) \stackrel{\tau}{\rightarrow} \mathcal{T}(M, X) \stackrel{\psi}{\rightarrow} M
$$

with $\tau$ idempotent pure and $\psi$ idempotent separating. We write $\mathcal{T}$ for $\mathcal{T}(M, X)$ : then the Squier complex $\mathrm{Sq}(\mathcal{P})$ of $\mathcal{P}$ is the 2 -complex constructed as follows.

- The vertex set is $\mathcal{T}$.

- The edge set consists of all 4-tuples $(p, l, r, q)$ with $p, q \in \mathcal{T}$ and $(l, r) \in R$. Such an edge will start at $p(l \rho \tau) q$ and end at $p(r \rho \tau) q$, so each edge corresponds to the application of a relation from $\mathcal{P}$ in $\mathcal{T}$. An edge path $\operatorname{in} \operatorname{Sq}(\mathcal{P})$ therefore corresponds to a succession of such applications.

- The 2-cells correspond to applications of non-overlapping relations, and so a 2-cell is attached along every edge path of the form:

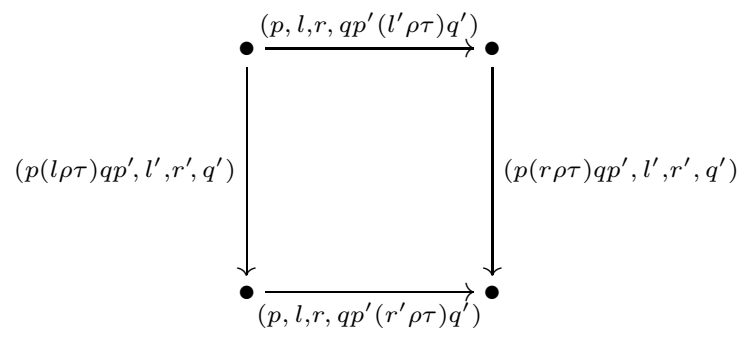


This attachment of 2-cells makes the two edge paths between $p(l \rho \tau) q p^{\prime}\left(l^{\prime} \rho \tau\right) q^{\prime}$ and $p(r \rho \tau) q p^{\prime}\left(r^{\prime} \rho \tau\right) q^{\prime}$ homotopic in $\operatorname{Sq}(\mathcal{P})$.

Proposition 4.1. The fundamental groupoid $\pi(S q(\mathcal{P}), \mathcal{T})$ is pseudoregular and monoidal.

Proof. The actions of $\mathcal{T}$ on single edges in $\mathrm{Sq}(\mathcal{P})$ given by

$$
t \triangleright(p, l, r, q)=(t p, l, r, q) \text { and }(p, l, r, q) \triangleleft t=(p, l, r, q t)
$$

induce a pseudoregular structure on the fundamental groupoid. The 2-cells of $\mathrm{Sq}(\mathcal{P})$ ensure that, if $\alpha$ and $\beta$ are the homotopy classes of edge-paths of length 1 in $\operatorname{Sq}(\mathcal{P})$, then $\alpha * \beta=\alpha \circledast \beta$, and a straightforward induction extends this to arbitrary edge-paths.

It will be convenient in what follows to describe operations in the fundamental groupoid $\pi(\mathrm{Sq}(\mathcal{P}), \mathcal{T})$ as being performed on edge-paths in $\mathrm{Sq}(\mathcal{P})$ rather than on fixed-end-point homotopy classes.

Let $e \in E(\mathcal{T})$. Then $\operatorname{star}_{e}^{\bowtie}(\pi(\mathrm{Sq}(\mathcal{P}), \mathcal{T}))$ has vertex set $K_{e}=\{a \in \mathcal{T}: a \psi=e\}$, which we recognise as one of the groups that make up the kernel of $\psi$.

Lemma 4.2. Let $e \in E(\mathcal{T})$. Then $\left(\operatorname{star}_{e}^{\bowtie}(\pi(\mathrm{Sq}(\mathcal{P}), \mathcal{T})), *\right)$ is a group.

Proof. The set $\operatorname{star}_{e}^{\bowtie}(\pi(\operatorname{Sq}(\mathcal{P}), \mathcal{T}))$ is a monoid under the operation $*$, and for $\alpha \in$ $\operatorname{star}_{e}^{\bowtie}(\pi(\operatorname{Sq}(\mathcal{P}), \mathcal{T}))$ we define

$$
\alpha^{*}=(\alpha \mathbf{r})^{-1} \triangleright \alpha^{\circ} \triangleleft(\alpha \mathbf{d})^{-1},
$$

where a superscript ${ }^{-1}$ denotes the inverse in the inverse monoid $\mathcal{T}$ and a superscript ${ }^{\circ}$ denotes the inverse in the groupoid $\mathrm{Sq}(\mathcal{P})$. Now

$$
\begin{aligned}
\alpha * \alpha^{*} & =\alpha *\left((\alpha \mathbf{r})^{-1} \triangleright \alpha^{\circ} \triangleleft(\alpha \mathbf{d})^{-1}\right) \\
& =\left(\alpha \triangleleft(\alpha \mathbf{r})^{-1}\left(\alpha^{\circ} \mathbf{d}\right)(\alpha \mathbf{d})^{-1}\right) \circ\left((\alpha \mathbf{r})(\alpha \mathbf{r})^{-1} \triangleright \alpha^{\circ} \triangleleft(\alpha \mathbf{d})^{-1}\right) \\
& =\left(\alpha \triangleleft(\alpha \mathbf{r})^{-1}(\alpha \mathbf{r})(\alpha \mathbf{d})^{-1}\right) \circ\left((\alpha \mathbf{r})(\alpha \mathbf{r})^{-1} \triangleright \alpha^{\circ} \triangleleft(\alpha \mathbf{d})^{-1}\right) .
\end{aligned}
$$

Since $\alpha \in \operatorname{star}_{e}^{\bowtie}(\pi(\operatorname{Sq}(\mathcal{P}), \mathcal{T}))$ we have $\alpha \mathbf{d}=e$, and since $\alpha \mathbf{r} \in K_{e}$ and $K_{e}$ is a subgroup of $\mathcal{T}$ with identity $e$, then $(\alpha \mathbf{r})^{-1}(\alpha \mathbf{r})=e=(\alpha \mathbf{r})(\alpha \mathbf{r})^{-1}$. So

$$
\alpha * \alpha^{*}=(\alpha \triangleleft e) \circ\left(e \triangleright \alpha^{\circ} \triangleleft e\right)=\alpha \circ \alpha^{\circ}=1_{e} .
$$

Similarly $\alpha^{*} * \alpha=1_{e}$, and $\operatorname{star}_{e}^{\bowtie}(\pi(\operatorname{Sq}(\mathcal{P}), \mathcal{T}))$ is a group.

Since we shall be working exclusively in the groupoid $\pi(\operatorname{Sq}(\mathcal{P}), \mathcal{T})$ hereon, we shall abbreviate $\operatorname{star}_{e}^{\bowtie}(\pi(\mathrm{Sq}(\mathcal{P}), \mathcal{T}))$ to $\operatorname{star}_{e}^{\bowtie}$.

Lemma 4.3. Suppose that $(e p, l, r, q e) \in \operatorname{star}_{e}^{\bowtie}$ and set $h=l \rho \tau$ and $k=r \rho \tau$. Then ephqe $=e$ and

$$
(e p)(h q e)(e p)=e e p=e p \quad \text { and } \quad(h q e)(e p)(h q e)=h q e e=h q e .
$$

Therefore ep $=(h q e)^{-1}$ in $\mathcal{T}$ and $(e p, l, r, q e)=\left(e q^{-1} h^{-1}, l, r, q e\right)$ : moreover $e=$ $e q^{-1} h^{-1} h q e$ and so $e \leqslant q^{-1} h^{-1} h q$.

Lemma 4.4. A path $\alpha \in \operatorname{star}_{e}^{\bowtie}$ can be written as a product of single edges in the group $\left(\operatorname{star}_{e}^{\bowtie}, *\right)$. Hence $\operatorname{star}_{e}^{\bowtie}$ is generated by the subset $\Sigma_{e}^{\bowtie}$ of homotopy classes of single edges in $\operatorname{star}_{e}^{\bowtie}$, and these classes are represented by edges of the form $\lambda_{l, r, q}^{e}=$ $\left(e q^{-1}\left(l^{-1}\right) \rho \tau, l, r, q e\right)$ with $e \leqslant q\left(\left(l^{-1} l\right) \rho \tau\right) q$. 
Proof. The vertex set of the connected component of $\operatorname{Sq}(\mathcal{P})$ that contains $e$ is the group $K_{e}$ (with identity $e$ ), and for a path $\alpha$ in this component we define

$$
\alpha \lambda=(\alpha \mathbf{d})^{-1} \triangleright \alpha \triangleleft e .
$$

If $\alpha=(p, l, r, q)$ is a single edge and $h=l \rho \tau$, then

$$
\alpha \lambda=\left(q^{-1} h^{-1} p^{-1} p, l, r, q e\right)=\left(e q^{-1} h^{-1} p^{-1} p, l, r, q e\right)
$$

since $p h q \in K_{e}$. Then by Lemma4.3, we have $e q^{-1} h^{-1} p^{-1} p=(h q e)^{-1}$ and so

$$
\alpha \lambda=\left(e q^{-1} h^{-1}, l, r, q e\right)=\lambda_{l, r, q}^{e},
$$

and $(\alpha \lambda) \mathbf{d}=e q^{-1} h^{-1} h q e=e$.

Now if $\alpha=\alpha_{1} \circ \alpha_{2}$ then

$$
\begin{aligned}
\alpha \lambda & =\left(\alpha_{1} \mathbf{d}\right)^{-1} \triangleright\left(\alpha_{1} \circ \alpha_{2}\right) \triangleleft e \\
& =\left(\left(\alpha_{1} \mathbf{d}\right)^{-1} \triangleright \alpha_{1} \triangleleft e\right) \circ\left(\left(\alpha_{1} \mathbf{d}\right)^{-1} \triangleright \alpha_{2} \triangleleft e\right) \\
& =\alpha_{1} \lambda \circ\left(\left(\alpha_{1} \mathbf{d}\right)^{-1} \triangleright \alpha_{2} \triangleleft e\right),
\end{aligned}
$$

and

$$
\alpha_{1} \lambda * \alpha_{2} \lambda=\left(\alpha_{1} \lambda \triangleleft e\right) \circ\left(\left(\alpha_{1} \mathbf{d}\right)^{-1}\left(\alpha_{1} \mathbf{r}\right) e\left(\alpha_{2} \mathbf{d}\right)^{-1} \triangleright \alpha_{2} \triangleleft e\right) .
$$

But $\alpha_{1} \mathbf{r}=\alpha_{2} \mathbf{d} \in K_{e}$ and so $\left(\alpha_{1} \mathbf{d}\right)^{-1}\left(\alpha_{1} \mathbf{r}\right) e\left(\alpha_{2} \mathbf{d}\right)^{-1}=\left(\alpha_{1} \mathbf{d}\right)^{-1}$ and therefore $\alpha \lambda=$ $\alpha_{1} \lambda * \alpha_{2} \lambda$. The Lemma then follows easily by induction on the length of a path.

Now suppose that a path $\alpha$ with $\alpha \mathbf{d}=e$ is a composition $\alpha=\alpha_{1} \circ \alpha_{2}$ and that $\beta$ is the path

$$
\beta=\alpha_{1} \circ \gamma \circ \gamma^{\circ} \circ \alpha_{2}
$$

for some path $\gamma$. Then

$$
\beta \lambda=\alpha_{1} \lambda * \gamma \lambda * \gamma^{\circ} \lambda * \alpha_{2} \lambda .
$$

Now if $x=\gamma \mathbf{d}$ and $y=\gamma \mathbf{r}$ then

$$
\begin{aligned}
\gamma \lambda * \gamma^{\circ} \lambda & =\left(x^{-1} \triangleright \gamma \triangleleft e\right) *\left(y^{-1} \triangleright \gamma^{\circ} \triangleleft e\right) \\
& =\left(x^{-1} \triangleright \gamma \triangleleft e\right) \circ\left(x^{-1} y \triangleright y^{-1} \triangleright \gamma^{\circ} \triangleleft e\right) \\
& =\left(x^{-1} \triangleright \gamma \triangleleft e\right) \circ\left(x^{-1} \triangleright \gamma^{\circ} \triangleleft e\right) \\
& =x^{-1} \triangleright\left(\gamma \circ \gamma^{\circ}\right) \triangleleft e \\
& =x^{-1} \triangleright 1_{x} \triangleleft e=1_{e} .
\end{aligned}
$$

Hence if $\alpha$ and $\beta$ are paths differing by a cancelling pair of edges in $\operatorname{Sq}(\mathcal{P})$ then $\alpha \lambda=\beta \lambda$ in the group $\left(\operatorname{star}_{e}^{\bowtie}, *\right)$.

Now consider a $2-$ cell in the component of $\mathrm{Sq}(\mathcal{P})$ containing $e$ :

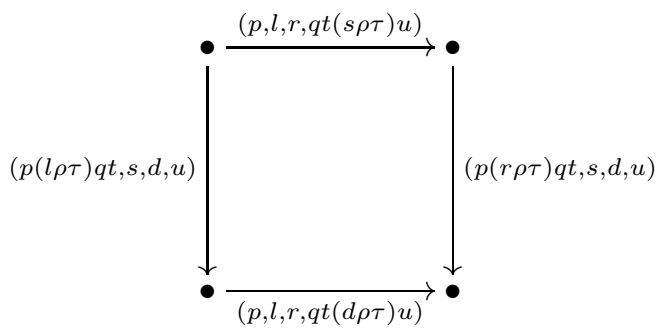


with

$$
\begin{aligned}
\alpha & =(p, l, r, q t(s \rho \tau) u), \beta=(p(r \rho \tau) q t, s, d, u), \gamma=(p(l \rho \tau) q t, s, d, u), \\
\delta & =(p, l, r, q t(d \rho \tau) u) .
\end{aligned}
$$

Then by Lemma4.3, $\alpha \lambda=\lambda_{l, r, q t(s \rho \tau) u}^{e}, \beta \lambda=\lambda_{s, d, u}^{e}=\gamma \lambda$ and $\delta \lambda=\lambda_{l, r, q t(d \rho \tau) u}^{e}$. Hence path homotopy induced by the above $2-$ cell in $\operatorname{Sq}(\mathcal{P})$ is equivalent to the relation

$$
\lambda_{l, r, v(s \rho \tau) u}^{e} * \lambda_{s, d, u}^{e}=\lambda_{s, d, u}^{e} * \lambda_{l, r, v(d \rho \tau) u}^{e}
$$

(where $v=q t$ above). These considerations show that:

Proposition 4.5. Given $e \in E(\mathcal{T}), q \in \mathcal{T}$ and $(l, r) \in \mathcal{R}$ with $e \leqslant q^{-1}\left(\left(l^{-1} l\right) \rho \tau\right) q$, we set $\lambda_{l, r, q}^{e}=\left(e q^{-1}\left(l^{-1} \rho \tau\right), l, r, q e\right)$. Then the following are a set of defining relations for the group $\left(\operatorname{star}_{e}^{\bowtie}, *\right)$ on the generating set $\Sigma_{e}^{\bowtie}$ :

$$
\lambda_{l, r, v(s \rho \tau) u}^{e} * \lambda_{s, d, u}^{e}=\lambda_{s, d, u}^{e} * \lambda_{l, r, v(d \rho \tau) u}^{e} .
$$

4.1. A crossed module from an inverse monoid presentation. As in Example 1.3, we regard $\mathcal{T}$ as a groupoid $\overrightarrow{\mathcal{T}}$ (although we shall drop the arrow superscript hereon) with vertex set $E=E(\mathcal{T})$, and we define

$$
S^{\bowtie}=\bigsqcup_{e \in E} \operatorname{star}_{e}^{\bowtie}(\pi(\operatorname{Sq}(\mathcal{P}), \mathcal{T})) .
$$

Proposition 4.6. $S^{\bowtie} \stackrel{\mathrm{r}}{\rightarrow} \mathcal{T} \rightrightarrows E$ is a crossed module of groupoids.

Proof. By Lemma 4.2, each $\operatorname{star}_{e}^{\bowtie}$ is a group, and so $S^{\bowtie}$ is a disjoint union of groups indexed by $E$, and is a groupoid with vertex set $E$. Then $\mathbf{r}$ is a groupoid homomorphism, and is the identity on $E$.

An action of $\mathcal{T}$ on $S^{\bowtie}$ is defined using the actions in the pseudoregular groupoid $\pi(\operatorname{Sq}(\mathcal{P}), \mathcal{T})$ as follows: for $w \in \mathcal{T}$ and $\alpha \in \operatorname{star}_{w w^{-1}}^{\bowtie}$ we define

$$
\alpha^{w}=w^{-1} \triangleright \alpha \triangleleft w \in \operatorname{star}_{w^{-1} w}^{\bowtie} .
$$

Then CM1 holds, since $\left(\alpha^{w}\right) \mathbf{r}=\left(w^{-1} \triangleright \alpha \triangleleft w\right) \mathbf{r}=w^{-1}(\alpha \mathbf{r}) w$. For CM2, since the binary operations $*$ and $\circledast$ on $\pi(S q(\mathcal{P}), \mathcal{T})$ coincide by Proposition 4.1, then

$$
\alpha * \beta=\alpha \circledast \beta=\beta \circ(\alpha \triangleleft \beta \mathbf{r})
$$

and

$$
\beta * \alpha^{\beta \mathbf{r}}=\beta *\left((\beta \mathbf{r})^{-1} \triangleright \alpha \triangleleft \beta \mathbf{r}\right)=\beta \circ(\alpha \triangleleft \beta \mathbf{r}) .
$$

So $\alpha * \beta=\beta * \alpha^{\beta \mathbf{r}}$. Therefore $S^{\bowtie} \stackrel{\mathbf{r}}{\rightarrow} \mathcal{T} \rightrightarrows E$ is a crossed module of groupoids.

We shall now show that the crossed module $S^{\bowtie} \stackrel{\mathrm{r}}{\rightarrow} \mathcal{T} \rightrightarrows E$ is free, and give an explicit basis. To do this, we give a construction of a free crossed $\mathcal{T}(M, X)$-module directly from an inverse monoid presentation $\mathcal{P}=[X: \mathcal{R}]$ of $M$ and show that it is isomorphic to the one in Proposition 4.6

Suppose that $(l, r) \in \mathcal{R}$, and set $h=l \rho \tau$ and $k=r \rho \tau$. Then $h \psi=k \psi$ and so $\left(h^{-1} k\right) \psi \in$ $E(M)$. Since $\psi: \mathcal{T} \rightarrow M$ is idempotent separating, $\left(h^{-1} k\right) \psi=x \psi$ for a unique $x \in$ $E(\mathcal{T})$ with

$$
\left(h^{-1} k\right) \psi=\left(h^{-1} h\right) \psi=\left(k^{-1} k\right) \psi \quad \text { and } \quad\left(h k^{-1}\right) \psi=\left(h h^{-1}\right) \psi=\left(k k^{-1}\right) \psi .
$$


Hence $h^{-1} h=x=k^{-1} k$ and $h h^{-1}=k k^{-1}$. So for $x \in E(\mathcal{T})$ we define

$$
R_{x}=\left\{(l, r, x) \in \mathcal{R} \times E:\left(l^{-1} r\right) \rho \tau \psi \geqslant x \psi\right\},
$$

and consider the set $R=\bigsqcup_{x \in E} R_{x}$, along with the function $\omega: R \rightarrow \mathcal{T}$ which maps $(l, r, x) \mapsto x\left(\left(l^{-1} r\right) \rho \tau\right) x$. Then

$$
(l, r, x) \omega \mathbf{d}=x h^{-1} k x k^{-1} h x \text { and }(l, r, x) \omega \mathbf{r}=x k^{-1} h x h^{-1} k x,
$$

with

$$
((l, r, x) \omega \mathbf{d}) \psi=\left(x h^{-1} k x k^{-1} h x\right) \psi=x \psi=\left(x k^{-1} h x h^{-1} k x\right) \psi=((l, r, x) \omega \mathbf{r}) \psi .
$$

Since $\psi$ is idempotent separating, we conclude that $(l, r, x) \omega \mathbf{d}=(l, r, x) \omega \mathbf{r}$. The free crossed $\mathcal{T}$-module $C \stackrel{\partial}{\rightarrow} \mathcal{T} \rightrightarrows E(M)$ with basis $\omega$ is then constructed as in Proposition 1.6 .

Theorem 4.7. The crossed $\mathcal{T}$-module $S^{\bowtie} \stackrel{\mathbf{r}}{\rightarrow} \mathcal{T} \rightrightarrows E(M)$ is isomorphic to the free crossed $\mathcal{T}$-module $C \stackrel{\partial}{\rightarrow} \mathcal{T} \rightrightarrows E(M)$.

Proof. For $(l, r, x) \in R$, we retain the notation $h=l \rho \tau$ and $k=r \rho \tau$. We define $\nu: R \rightarrow$ $S^{\bowtie}$ by $(l, r, x) \mapsto\left(x h^{-1}, l, r, x\right)$. We then have $\left(x h^{-1}, l, r, x\right) \mathbf{d}=x h^{-1} h x=x$, and so $(l, r, x) \nu \in \operatorname{star}_{x}^{\bowtie}$. Moreover,

$$
(l, r, x) \nu \mathbf{r}=\left(x h^{-1}, l, r, x\right) \mathbf{r}=x h^{-1} k x=(l, r, x) \omega .
$$

Therefore $\nu \mathbf{r}=\omega$ and by freeness of $C \stackrel{\partial}{\rightarrow} \mathcal{T} \rightrightarrows E(M)$ there exists a crossed module morphism $\eta: C \rightarrow S^{\bowtie}$ mapping

$$
(l, r, u) \mapsto\left(u^{-1} h^{-1}, l, r, u\right) \in \operatorname{star}^{\bowtie}\left(u^{-1} u\right),
$$

where $\left(h^{-1} k\right) \psi \geqslant\left(u u^{-1}\right) \psi$ and $h^{-1} h=k^{-1} k \geqslant u u^{-1}$. We claim that $\eta$ is an isomorphism, and we verify this by constructing its inverse.

We define $\mu: \Sigma_{e}^{\bowtie} \rightarrow C_{e}$ by $\mu: \lambda_{l, r, q}^{e} \mapsto(l, r, q e)$, where $\lambda_{l, r, q}^{e}$ is defined in Proposition 4.5. We consider the effect of this map on a defining relation

$$
\lambda_{l, r, v(s \rho \tau) u}^{e} * \lambda_{s, d, u}^{e}=\lambda_{s, d, u}^{e} * \lambda_{l, r, v(d \rho \tau) u}^{e} .
$$

as given in Proposition 4.5 We set $g=s \rho \tau$ and $t=d \rho \tau$. Then

$$
\lambda_{l, r, v g u}^{e} \stackrel{\mu}{\mapsto}(l, r, v g u e), \lambda_{s, d, u}^{e} \stackrel{\mu}{\mapsto}(s, d, u e) \text {, and } \lambda_{l, r, v t u}^{e} \stackrel{\mu}{\mapsto}(l, r, v t u e) \text {. }
$$

In the group $C_{e}$ we have

$$
(s, d, u e)^{-1}(l, r, \text { vgue })(s, d, u e)=\left(l, r, v_{\text {gueu }}{ }^{-1} g^{-1} \text { tue }\right) .
$$

Now $g \psi=t \psi$ and so

$$
\left[(\text { gue })(g u e)^{-1}\right] \psi=\left[(\text { tue })(\text { tue })^{-1}\right] \psi .
$$

Since $\psi$ is idempotent separating, $(g u e)(g u e)^{-1}=(t u e)(t u e)^{-1}$ and therefore

$$
\text { vgue } u^{-1} g^{-1} \text { tue }=v(\text { gue })(\text { gue })^{-1} \text { tue }=v(\text { tue })(\text { tue })^{-1}(\text { tue })=v t u e .
$$

So in $C_{e}$ we have

$$
(s, d, u e)^{-1}(l, r, v g u e)(s, d, u e)=(l, r, v t u e)
$$

and $\mu$ induces a homomorphism $\operatorname{star}_{e}^{\bowtie} \rightarrow C_{e}$ that is the inverse of $\eta$.

As a module for the groupoid $\vec{M}$, we see by Proposition 1.7 that $\left(S^{\bowtie}\right)^{a b}$ is free, with basis function $(l, r, u) \mapsto \overline{\left(u^{-1} l^{-1}, l, r, u\right)}$. However, we can say more. 
Proposition 4.8. $\left(S^{\bowtie}\right)^{a b}$ is the free $M$-module on the $E(M)-$ set $\mathcal{Z}$ in which $Z_{e}=$ $\left\{(l, r) \in R:\left(l^{-1} r\right) \rho \tau \psi=e \psi\right\}$.

Proof. The groupoid action of $\vec{M}$ extends to one of $M$. If $\alpha \in \operatorname{star}_{e}^{\bowtie}$ with image $\bar{\alpha} \in$ $\left(\operatorname{star}_{e}^{\bowtie}\right)^{a b}$, and $m \in M$ with $m=w \psi$, then we define

$$
\bar{\alpha} \triangleleft m=\overline{w^{-1} \triangleright \alpha \triangleleft w} .
$$

As a component of the free $\vec{M}$-module $\left(S^{\bowtie}\right)^{a b}$, the group $\left(\operatorname{star}_{e}^{\bowtie}\right)^{a b}$ is the free abelian group with basis

$$
Y_{e}=\left\{(l, r, m):\left(l^{-1} r\right) \rho \tau \psi \geqslant m m^{-1}, m^{-1} m=e\right\} .
$$

which is the correct basis for $\left(S^{\bowtie}\right)^{a b}$ as the free $M$-module on the $E(M)$-set $\mathcal{Z}$.

4.2. A presentation for the relation module. From an inverse monoid presentation $\mathcal{P}=$ $[X: \mathcal{R}]$ of an inverse monoid $M$ we have now constructed a free crossed module $S^{\bowtie} \stackrel{\mathbf{r}}{\rightarrow}$ $\mathcal{T} \rightrightarrows E$ and for each $e \in E$ we have a crossed module of groups $\operatorname{star}_{e}^{\bowtie} \stackrel{\mathbf{r}}{\rightarrow} K_{e}$. Since $K_{e}$ is the vertex set of the component of $\operatorname{Sq}(\mathcal{P})$ containing $e$, the map $\mathbf{r}: \operatorname{star}_{e}^{\bowtie} \rightarrow K_{e}$ is surjective. By Propositions 2.4 and 4.1 .

$$
\pi_{e}^{\bowtie}=\left\{\alpha \in \operatorname{star}_{e}^{\bowtie}: \alpha \mathbf{r}=e\right\}
$$

is abelian and we have a short exact sequence of groups

$$
0 \rightarrow \pi_{e}^{\bowtie} \rightarrow \operatorname{star}_{e}^{\bowtie} \rightarrow K_{e} \rightarrow 1 .
$$

Lemma 4.9. Each group $K_{e}$ is free, the sequence (4.2) splits and, $\operatorname{star}_{e}^{\bowtie}$ and $\pi_{e}^{\bowtie} \times K_{e}$ are isomorphic groups.

Proof. The group $K_{e}$ is a subgroup of $\mathcal{T}$ and the maximum group image map $\sigma: \mathcal{T} \rightarrow$ $F(X)$ is idempotent pure. Its restriction $\sigma: K_{e} \rightarrow F(X)$ therefore has trivial kernel and so $K_{e}$ is isomorphic to a subgroup of a free group and is free. By (1.2), $K_{e}$ acts trivially on $\pi_{e}^{\bowtie}$ and so the splitting of the sequence (4.2) induces an isomorphism $\operatorname{star}_{e}^{\bowtie} \cong \pi_{e}^{\bowtie} \times K_{e}$.

Theorem 4.10. Let $\mathcal{P}$ be an inverse monoid presentation of an inverse monoid $M$. There exists a short exact sequence of $M-$ modules

$$
0 \rightarrow \bigsqcup_{e \in E(M)} \pi_{e}^{\bowtie} \rightarrow\left(S^{\bowtie}\right)^{a b} \stackrel{\overline{\mathbf{r}}}{\rightarrow} \mathcal{K} \rightarrow 0
$$

in which $\left(S^{\bowtie}\right)^{a b}$ is a free $M$-module and $\overline{\mathbf{r}}$ is induced by $S^{\bowtie} \stackrel{\mathbf{r}}{\rightarrow} K$.

Proof. The $M$-module structure on $\bigsqcup_{e \in E(M)} \pi_{e}^{\bowtie}$ is given by Proposition 2.4 that on $\left(S^{\bowtie}\right)^{a b}$ by Proposition 4.8, and that on $\mathcal{K}$ by Lemma 1.5. Lemma 4.9 gives us, for each $e \in E(M)$, a short exact sequence of abelian groups

$$
0 \rightarrow \pi_{e}^{\bowtie} \rightarrow \operatorname{star}_{e}^{\bowtie} \stackrel{\bar{\partial}}{\rightarrow} K_{e}^{a b} \rightarrow 0
$$

and these assemble into the sequence (4.3). It remains to check that $\overline{\mathbf{r}}$ is then a map of $M$-modules. 
Let $\alpha \in \operatorname{star}_{e}^{\bowtie}$ with image $\bar{\alpha} \in\left(S^{\bowtie}\right)^{a b}$, and let $m \in M$ with $m m^{-1}=e$. Then the action of $m$ on $\bar{\alpha}$ is defined by lifting $m$ to $\mathcal{T}$ and acting on $\alpha$ in the crossed module $S^{\bowtie} \stackrel{\text { r }}{\rightarrow} \mathcal{T}$ :

$$
\bar{\alpha} \triangleleft m=\overline{t^{-1} \triangleright \alpha \triangleleft t}
$$

where $t \psi=m$. Hence

$$
(\bar{\alpha} \triangleleft m) \overline{\mathbf{r}}=\overline{\left(t^{-1} \triangleright \alpha \triangleleft t\right) \mathbf{r}}=\overline{t^{-1}(\alpha \mathbf{r}) t} \in U_{m^{-1} m}^{a b} .
$$

But $\bar{\alpha} \mathbf{r}=\overline{\alpha \mathbf{r}}$ and $\overline{\alpha \mathbf{r}} \triangleleft m=\overline{t^{-1}(\alpha \mathbf{r}) t}$.

Acknowledgements. A version of these results is presented in the second author's PhD thesis at Heriot-Watt University, Edinburgh. The generous financial support of a $\mathrm{PhD}$ Scholarship from the Carnegie Trust for the Universities of Scotland is duly and gratefully acknowledged.

\section{REFERENCES}

[1] T. Brough, Word problem languages for free inverse monoids. In Descriptional complexity of formal systems, Lecture Notes in Comput. Sci. 10952, Springer (2018) 24-36.

[2] R. Brown and J. Huebschmann, Identities among relations. In Low Dimensional Topology, R. Brown and T.L. Thickstun (eds.), London Math. Soc. Lect. Notes 48, Cambridge University Press (1982) 153-202.

[3] R. Brown and N.D. Gilbert, Automorphism structures for crossed modules and algebraic models of 3-types. Proc. London Math. Soc. (3) 59 (1989) 51-73.

[4] R. Brown, Possible connections between whiskered categories and groupoids, Leibniz algebras, automorphism structures and local-to-global questions. Journal of Homotopy and Related Structures, vol. 1(1), 2010, pp.1-13.

[5] R. Brown, P.J. Higgins, and R. Sivera, Nonabelian algebraic topology. Filtered spaces, crossed complexes, cubical homotopy groupoids. EMS Tracts in Mathematics 15. European Mathematical Society (2011).

[6] R. Cremanns and F. Otto, For groups the property of having finite derivation type is equivalent to the homological finiteness condition $F P_{3}$. J. Symb. Comput. 22 (1996) 155-177.

[7] R.H. Crowell, The Derived Module of a Homomorphism. Advances in Mathematics 6 (1971) 210-238.

[8] A. Cutting and A. Solomon, Remarks concerning finitely generated semigroups having regular sets of unique normal forms. J. Aust. Math. Soc. 70 (2001) 293-309.

[9] N.D. Gilbert, Monoid presentations and associated groupoids. Internat. J. Algebra Comput. 8 (1998) 141152.

[10] N.D. Gilbert, Derivations and relation modules for inverse semigroups. Algebra Discrete Math. 12 (2011) 1-19.

[11] N.D. Gilbert and E.A. McDougall, Groupoids and the algebra of rewriting in group presentations. Preprint, arxiv.org/abs/1901.04348 (2019).

[12] P.J. Higgins, Notes on categories and groupoids. Van Nostrand Reinhold Math. Stud. 32 (1971). Reprinted electronically at www.tac.mta.co/tac/reprints/articles/7/7tr7.pdf .

[13] J.M. Howie, Fundamentals of Semigroup Theory. London Math. Soc. Monographs, Oxford University Press (1997).

[14] V. Kilibarda, On the algebra of semigroup diagrams. Internat. J. Algebra Comput. 7 (1997) 313-338.

[15] H. Lausch, Cohomology of inverse semigroups. J. Algebra 35 (1975) 273-303.

[16] M.V. Lawson, Inverse Semigroups. World Scientific (1998)

[17] R. Lavendhomme and J.R. Roisin, Cohomologie non abélienne de structures algébriques. J. Algebra 67 383-414 (1980).

[18] S.W. Margolis and J.C. Meakin, E-unitary inverse monoids and the Cayley graph of a group presentation. J. Pure Appl. Algebra 58 (1989) 45-76.

[19] J.C. Meakin, Groups and semigroups: connections and contrasts. In Groups St Andrews 2005 Vol. 2, C.M.Campbell et al. Eds. London Math. Soc. Lect. Notes 340, Cambridge University Press (2007) 357-400.

[20] W.R. Nico, On the regularity of semidirect products. J. Algebra 80 29-36 (1983). (1970) 824-827.

[21] S.J. Pride, Low-dimensional homotopy theory for monoids I. Internat. J. Algebra Comput. 5 (1995) 631-649.

[22] S.J. Pride, Low-dimensional homotopy theory for monoids II: groups. Glasg. Math. J. 41 (1999) 1-11.

[23] N.R. Reilly and H.E. Scheiblich, Congruences on regular semigroups. Pacific J. Math. 23 (1967) 349-360.

[24] B.M. Schein, Free inverse semigroups are not finitely presentable. Acta Math. Hungarica 26 (1975) 41-52. 
[25] C.C. Squier, Word problems and a homological finiteness condition for monoids. J. Pure Appl. Algebra 49 (1987) 201-216.

[26] C.C. Squier, F. Otto, and Y. Kobayashi, A finiteness condition for rewriting systems. Theoret. Comput. Sci. 131 (1994) 271-294.

[27] J.B. Stephen, Presentations of inverse monoids. J.Pure Appl. Algebra 63 (1990) 81-112. 